\title{
ON BASE AND TURYN SEQUENCES
}

\author{
C. KOUKOUVINOS, S. KOUNIAS, AND K. SOTIRAKOGLOU
}

\begin{abstract}
Base sequences of lengths $n+1, n+1, n, n$ are constructed for all decompositions of $4 n+2$ into four squares for $n=19, \ldots, 24$. The construction is achieved through an algorithm which is also presented. It is proved through an exhaustive search that Turyn sequences do not exist for $n=18, \ldots, 27$; since Turyn sequences cannot exist for $n=28$ or 29 , the first unsettled case is $n=30$.
\end{abstract}

\section{INTRODUCTION}

Given the sequence $A=\left\{a_{1}, \ldots, a_{n}\right\}$ of length $n$, the nonperiodic autocorrelation function $N_{A}(s)$ is defined as

$$
N_{A}(s)=\sum_{i=1}^{n-s} a_{i} a_{i+s}, \quad s=0,1, \ldots, n-1 .
$$

If $A(z)=a_{1}+a_{2} z+\cdots+a_{n} z^{n-1}$ is the polynomial associated with the sequence $A$, then

(2) $A(z) A\left(z^{-1}\right)=\sum_{i=1}^{n} \sum_{j=1}^{n} a_{i} a_{j} z^{i-j}=N_{A}(0)+\sum_{s=1}^{n-1} N_{A}(s)\left(z^{s}+z^{-s}\right), \quad z \neq 0$.

If $A^{*}=\left\{a_{n}, \ldots, a_{1}\right\}$ is the reversed sequence, then

$$
A^{*}(z)=z^{n-1} A\left(z^{-1}\right) \text {. }
$$

Base and Turyn sequences are finite sequences, with zero autocorrelation function, useful in constructing orthogonal designs and Hadamard matrices [3], in communications engineering [5], etc.

Definition 1. The four sequences $X, Y, Z, W$ of length $n$, with entries 0 , $1,-1$, are called $T$-sequences if

(i) $\left|x_{i}\right|+\left|y_{i}\right|+\left|z_{i}\right|+\left|w_{i}\right|=1, \quad i=1, \ldots, n$,

(ii) $N_{X}(s)+N_{Y}(s)+N_{Z}(s)+N_{W}(s)= \begin{cases}0, & s=1, \ldots, n-1, \\ n, & s=0 .\end{cases}$

Received April 14, 1989; revised September 21, 1989.

1980 Mathematics Subject Classification (1985 Revision). Primary 62K05, 62K10; Secondary 05B20.

Key words and phrases. Associate polynomial, algorithm, autocorrelation, cross-correlation, construction.

This research was partially supported by grant No. 87ED73 from the Secretariat for Research and Technology of Greece. 
Yang [6] gives another name for $T$-sequences and calls them four-symbol $\delta$-codes; he also calls the quadruple $Q=X+Y, R=X-Y, S=Z+W$, $T=Z-W$ a regular $\delta$-code of length $n$, where $X, Y, Z, W$ are $T$ sequences of length $n$.

If Williamson type matrices of size $w$ exist, and $T$-sequences of length $n$ exist, then Hadamard matrices of size $4 n \mathrm{w}$ can be constructed (Cooper and (Seberry) Wallis [2]).

If $X(z), Y(z), Z(z), W(z)$ are the associated polynomials, then from Definition 1 and (2) we see that (ii) in (4) can be replaced by
$X(z) X\left(z^{-1}\right)+Y(z) Y\left(z^{-1}\right)+Z(z) Z$
$(z) Z\left(z^{-1}\right)+W(z) W\left(z^{-1}\right)=n$
$z \neq 0$.

In $\S 2$ we develop an algorithm and we construct base sequences of lengths $n+1$, $n+1, n, n$ for $n=19, \ldots, 24$ for all decompositions of $4 n+2$ into four squares. These are given in Table 1 . In $\S 3$ we use an exhaustive search to prove that Turyn sequences of lengths $n+1, n+1, n, n$, do not exist for $n=18, \ldots, 27$.

\section{BASE SEQUENCES}

Definition 2. The four sequences $A, B, C, D$ of lengths $n+p, n+p, n$, $n$, with entries $+1,-1$, are called base sequences if

$$
\begin{aligned}
& N_{A}(s)+N_{B}(s)+N_{C}(s)+N_{D}(s)= \begin{cases}0, & s=1, \ldots, n-1, \\
4 n+2 p, & s=0,\end{cases} \\
& N_{A}(s)+N_{B}(s)=0, \quad s=n, \ldots, n+p-1 .
\end{aligned}
$$

Equivalently, (6) can be replaced by (see (2))

$$
\begin{array}{r}
A(z) A\left(z^{-1}\right)+B(z) B\left(z^{-1}\right)+C(z) C\left(z^{-1}\right)+D(z) D\left(z^{-1}\right)=4 n+2 p, \\
z \neq 0,
\end{array}
$$

where $A(z), B(z), C(z), D(z)$ are the associated polynomials. Base sequences of lengths $n+p, n+p, n, n$, whenever $p=1$, are denoted by $\mathrm{BS}(2 n+1)$.

From (7), and for $p=1$, if we set $z=1$, we obtain

$$
a^{2}+b^{2}+c^{2}+d^{2}=4 n+2,
$$

where $a, b, c, d$ are the sum of the elements of $A, B, C, D$, respectively. BS $(2 n+1)$ for all decompositions of $4 n+2$ into four squares for $n=1, \ldots, 18$ are given in [1, 4]. Also, BS $(2 n+1)$ for $n=19,20, \ldots, 23,25,26,29$ and $n=2^{a} 10^{b} 26^{c}$ (Golay numbers), $a, b, c$ nonnegative integers, are given in Yang [7].

In Table 1 we give $\mathrm{BS}(2 n+1)$ for all decompositions of $4 n+2$ into four squares for $n=19, \ldots, 24$. 
Theorem 1. If $A=\left\{a_{1}, \ldots, a_{n+1}\right\}, B=\left\{b_{1}, \ldots, b_{n+1}\right\}, C=\left\{c_{1}, \ldots, c_{n}\right\}$, $D=\left\{d_{1}, \ldots, d_{n}\right\}$ are $\mathrm{BS}(2 n+1)$, then

$$
\begin{aligned}
a_{s}+b_{s}+a_{n+2-s}+b_{n+2-s} & \equiv \begin{cases}2 \bmod 4, & s=1, \\
0 \bmod 4, & s=2, \ldots,[(n+1) / 2],\end{cases} \\
c_{s}+d_{s}+c_{n+1-s}+d_{n+1-s} \equiv 0 \bmod 4, & s=1, \ldots,[n / 2] .
\end{aligned}
$$

Proof. We have

$$
\begin{aligned}
& N_{A}(s)+N_{B}(s)=\sum_{i=1}^{n+1-s}\left(a_{i} a_{i+s}+b_{i} b_{i+s}\right), \quad s=1, \ldots, n, \\
& N_{C}(s)+N_{D}(s)=\sum_{i=1}^{n-s}\left(c_{i} c_{i+s}+d_{i} d_{i+s}\right), \quad s=1, \ldots, n-1 .
\end{aligned}
$$

Then

$$
\begin{aligned}
& N_{A}(s)+N_{B}(s)+N_{C}(s)+N_{D}(s)= \sum_{i=1}^{n-s}\left(a_{i} a_{i+s}+b_{i} b_{i+s}+c_{i} c_{i+s}+d_{i} d_{i+s}\right) \\
&+a_{n+1} a_{n+1-s}+b_{n+1} b_{n+1-s}=0, \\
& s=1, \ldots, n-1,
\end{aligned}
$$

and since $x y \equiv(x+y-1) \bmod 4$ whenever $x, y= \pm 1$, we have from (10)

$$
\begin{aligned}
F(s):= & \sum_{i=1}^{n-s}\left(a_{i}+a_{i+s}+b_{i}+b_{i+s}+c_{i}+c_{i+s}+d_{i}+d_{i+s}\right) \\
& +a_{n+1}+a_{n+1-s}+b_{n+1}+b_{n+1-s} \equiv 2 \bmod 4, \quad s=1, \ldots, n-1,
\end{aligned}
$$

or

$$
\begin{aligned}
F(s)= & \sum_{i=1}^{n-s}\left(a_{i}+b_{i}+c_{i}+d_{i}\right)+\sum_{i=s+1}^{n}\left(a_{i}+b_{i}+c_{i}+d_{i}\right) \\
& +a_{n+1}+b_{n+1}+a_{n+1-s}+b_{n+1-s} \\
\equiv & 2 \bmod 4, \quad s=1, \ldots, n-1 .
\end{aligned}
$$

Now

$$
\begin{aligned}
F(s-1)-F(s) & =a_{s}+b_{s}+c_{s}+d_{s}+a_{n+2-s}+b_{n+2-s}+c_{n+1-s}+d_{n+1-s} \\
& \equiv 0 \bmod 4, \quad s=2, \ldots, n-1,
\end{aligned}
$$

and for $s=n-1$ we have from (11)

(13) $a_{1}+b_{1}+c_{1}+d_{1}+a_{n}+b_{n}+c_{n}+d_{n}+a_{n+1}+b_{n+1}+a_{2}+b_{2} \equiv 2 \bmod 4$.

Also, from

$$
N_{A}(n)+N_{B}(n)=a_{1} a_{n+1}+b_{1} b_{n+1}=0
$$

we have

$$
a_{1}+b_{1}+a_{n+1}+b_{n+1} \equiv 2 \bmod 4
$$


From (12)-(14) we obtain

$$
\begin{aligned}
& \left(a_{s}+b_{s}+a_{n+2-s}+b_{n+2-s}\right)+\left(c_{s}+d_{s}+c_{n+1-s}+d_{n+1-s}\right) \\
& \equiv 0 \bmod 4, \quad s=2, \ldots, n .
\end{aligned}
$$

If we set $n+2-s$ instead of $s$ in (15), then

$$
\begin{aligned}
& \left(a_{n+2-s}+b_{n+2-s}+a_{s}+b_{s}\right)+\left(c_{n+2-s}+d_{n+2-s}+c_{s-1}+d_{s-1}\right) \\
& \quad \equiv 0 \bmod 4, \quad s=2, \ldots, n .
\end{aligned}
$$

From (15) and (16) we have

$$
\begin{aligned}
c_{s-1} & +c_{s}+d_{s-1}+d_{s}+c_{n+1-s}+c_{n+2-s}+d_{n+1-s}+d_{n+2-s} \\
& \equiv 0 \bmod 4, \quad s=2, \ldots, n .
\end{aligned}
$$

For $n$ odd, (17) becomes

$$
c_{s}+c_{n+1-s}+d_{s}+d_{n+1-s} \equiv 0 \bmod 4, \quad s=2, \ldots, n,
$$

because with $s=(n+1) / 2$, (17) gives

$$
c_{(n-1) / 2}+c_{(n+3) / 2}+d_{(n-1) / 2}+d_{(n+3) / 2} \equiv 0 \bmod 4 ;
$$

then set $s=(n+1) / 2-1$, etc.

From (15) and (18) we have

$$
a_{s}+b_{s}+a_{n+2-s}+b_{n+2-s} \equiv 0 \bmod 4, \quad s=2, \ldots, n .
$$

If we set $n+1-s$ instead of $s$ in (15), then we have similarly

$$
\begin{aligned}
& a_{s}+a_{s+1}+b_{s}+b_{s+1}+a_{n+1-s}+b_{n+1-s}+a_{n+2-s}+b_{n+2-s} \\
& \quad \equiv 0 \bmod 4, \quad s=2, \ldots, n-1 .
\end{aligned}
$$

For $n$ even, using the same argument as before, (20) becomes

$$
a_{s}+b_{s}+a_{n+2-s}+b_{n+2-s} \equiv 0 \bmod 4, \quad s=2, \ldots, n-1,
$$

and from (15)

$$
c_{s}+d_{s}+c_{n+1-s}+d_{n+1-s} \equiv 0 \bmod 4, \quad s=2, \ldots, n-1 .
$$

Therefore, (14), (18), (19), (21), and (22) give the required result.

In (19) and (21) it is enough to take $s=2, \ldots,[(n+1) / 2]$, and in (18) and (22), $s=1, \ldots,[n / 2]$.

Before describing the algorithm, we need the following:

Given the sequence $E=\left\{e_{1}, \ldots, e_{n}\right\}$, we define the $m$ subsequences, for some $m=2,3, \ldots, n$ :

$$
\begin{aligned}
& E_{1}=\left\{e_{1}, e_{1+m}, \ldots, e_{1+s_{1} \cdot m}\right\} \quad \text { with } s_{1}=\left[\frac{n-1}{m}\right], \\
& E_{2}=\left\{e_{2}, e_{2+m}, \ldots, e_{2+s_{2} \cdot m}\right\} \quad \text { with } s_{2}=\left[\frac{n-2}{m}\right] \text {, } \\
& E_{m}=\left\{e_{m}, e_{2 m}, \ldots, e_{m+s_{m} \cdot m}\right\} \quad \text { with } s_{m}=\left[\frac{n-m}{m}\right],
\end{aligned}
$$


or

$$
E_{i}=\left\{e_{i}, e_{i+m}, \ldots, e_{i+s_{i} \cdot m}\right\} \quad \text { with } s_{i}=\left[\frac{n-i}{m}\right], \quad i=1, \ldots, m,
$$

with associated polynomials

$$
E_{i}(z)=\sum_{j=0}^{s_{i}} z^{j} \cdot e_{i+j \cdot m}, \quad i=1, \ldots, m .
$$

Then

$$
E(z)=E_{1}\left(z^{m}\right)+z E_{2}\left(z^{m}\right)+\cdots+z^{m-1} E_{m}\left(z^{m}\right)
$$

or

$$
E(z)=\sum_{i=1}^{m} z^{i-1} E_{i}\left(z^{m}\right)
$$

Theorem 2. If $A=\left\{a_{1}, \ldots, a_{n+1}\right\}, B=\left\{b_{1}, \ldots, b_{n+1}\right\}, C=\left\{c_{1}, \ldots, c_{n}\right\}$, $D=\left\{d_{1}, \ldots, d_{n}\right\}$ are $(1,-1)$-sequences of lengths $n+1, n+1, n, n$, then they are $\mathrm{BS}(2 n+1)$ if and only if for some $m=2, \ldots, n+1$

$$
\begin{aligned}
& \sum_{i=1}^{m}\left(A_{i}\left(z^{m}\right) A_{i}\left(z^{-m}\right)+B_{i}\left(z^{m}\right) B_{i}\left(z^{-m}\right)\right. \\
& \left.\quad+C_{i}\left(z^{m}\right) C_{i}\left(z^{-m}\right)+D_{i}\left(z^{m}\right) D_{i}\left(z^{-m}\right)\right)=4 n+2, \\
& \sum_{i=1}^{m-s}\left(A_{i}\left(z^{m}\right) A_{i+s}\left(z^{-m}\right)+B_{i}\left(z^{m}\right) B_{i+s}\left(z^{-m}\right)\right. \\
& \left.\quad+C_{i}\left(z^{m}\right) C_{i+s}\left(z^{-m}\right)+D_{i}\left(z^{m}\right) D_{i+s}\left(z^{-m}\right)\right) \\
& \quad+z^{m} \sum_{i=1}^{s}\left(A_{i+m-s}\left(z^{m}\right) A_{i}\left(z^{-m}\right)+B_{i+m-s}\left(z^{m}\right) B_{i}\left(z^{-m}\right)\right. \\
& \left.\quad+C_{i+m-s}\left(z^{m}\right) C_{i}\left(z^{-m}\right)+D_{i+m-s}\left(z^{m}\right) D_{i}\left(z^{-m}\right)\right)=0, \\
& \quad s=1, \ldots,[m / 2] .
\end{aligned}
$$

Proof. Writing $A(z), B(z), C(z), D(z)$ as in (23), and equating all coefficients of $z^{t}$ in (7), where $t \equiv s \bmod m$, we find the above relations (24) for $s=1, \ldots, m-1$. By setting $z^{-1}$ instead of $z$ in (24) we see that it is enough to take $s=1, \ldots,[\mathrm{m} / 2]$.

For $m=2$ we have

$$
\begin{aligned}
& A(z)=A_{1}\left(z^{2}\right)+z A_{2}\left(z^{2}\right), \\
& B(z)=B_{1}\left(z^{2}\right)+z B_{2}\left(z^{2}\right), \\
& C(z)=C_{1}\left(z^{2}\right)+z C_{2}\left(z^{2}\right), \\
& D(z)=D_{1}\left(z^{2}\right)+z D_{2}\left(z^{2}\right) .
\end{aligned}
$$


From (24) and (25) we conclude that there are four isomorphic transformations for the $\operatorname{BS}(2 n+1), A, B, C, D$, i.e.,

(i) interchange $A$ and $B$ and/or $C$ and $D$,

(ii) reverse one or more sequences,

(iii) negate one or more sequences,

(iv) negate alternate elements in all sequences.

Corollary 1. If $A, B, C, D$ are $\operatorname{BS}(2 n+1)$ and given $m \in\{2,3, \ldots, n+1\}$,

(i) $k_{i m}=\sum_{j \equiv i \bmod m} a_{j}, \quad r_{i m}=\sum_{j \equiv i \bmod m} b_{j}$,

$$
p_{i m}=\sum_{j \equiv i \bmod m} c_{j}, \quad q_{i m}=\sum_{j \equiv i \bmod m} d_{j},
$$

(ii) $K_{m}=\left\{k_{1 m}, \ldots, k_{m m}\right\}, \quad R_{m}=\left\{r_{1 m}, \ldots, r_{m m}\right\}$, $P_{m}=\left\{p_{1 m}, \ldots, p_{m m}\right\}, \quad Q_{m}=\left\{q_{1 m}, \ldots, q_{m m}\right\}$,

(iii) $N_{K}(s)=\sum_{i=1}^{m-s} k_{i m} k_{i+s, m}, \quad N_{R}(s)=\sum_{i=1}^{m-s} r_{i m} r_{i+s, m}$,

$$
N_{P}(s)=\sum_{i=1}^{m-s} p_{i m} p_{i+s, m}, \quad N_{Q}(s)=\sum_{i=1}^{m-s} q_{i m} q_{i+s, m},
$$

then for the given $m \in\{2,3, \ldots, n+1\}$,

$$
\begin{aligned}
& N_{K}(0)+N_{R}(0)+N_{P}(0)+N_{Q}(0) \\
&= k_{1 m}^{2}+\cdots+k_{m m}^{2}+r_{1 m}^{2}+\cdots+r_{m m}^{2} \\
&+p_{1 m}^{2}+\cdots+p_{m m}^{2}+q_{1 m}^{2}+\cdots+q_{m m}^{2} \\
&= 4 n+2, \\
& N_{K}(s)+N_{R}(s)+N_{P}(s)+N_{Q}(s)+N_{K}(m-s)+N_{R}(m-s) \\
&+N_{P}(m-s)+N_{Q}(m-s)=0, \quad s=1, \ldots,[m / 2] .
\end{aligned}
$$

Proof. If we set $z^{m}=1$, then $A_{i}\left(z^{m}\right), B_{i}\left(z^{m}\right), C_{i}\left(z^{m}\right), D_{i}\left(z^{m}\right)$ give

$$
A_{i}(1)=k_{i m}, \quad B_{i}(1)=r_{i m}, \quad C_{i}(1)=p_{i m}, \quad D_{i}(1)=q_{i m},
$$

and (24) becomes

$$
\begin{gathered}
\sum_{i=1}^{m}\left(k_{i m}^{2}+r_{i m}^{2}+p_{i m}^{2}+q_{i m}^{2}\right)=4 n+2, \\
\sum_{i=1}^{m-s}\left(k_{i m} k_{i+s, m}+r_{i m} r_{i+s, m}+p_{i m} p_{i+s, m}+q_{i m} q_{i+s, m}\right) \\
+\sum_{i=1}^{s}\left(k_{i m} k_{i+m-s, m}+r_{i m} r_{i+m-s, m}+p_{i m} p_{i+m-s, m}+q_{i m} q_{i+m-s, m}\right)=0,
\end{gathered}
$$


that is,

$$
\begin{gathered}
N_{K}(0)+N_{R}(0)+N_{P}(0)+N_{Q}(0)=4 n+2 \\
N_{K}(s)+N_{R}(s)+N_{P}(s)+N_{Q}(s)+N_{K}(m-s)+N_{R}(m-s) \\
+N_{P}(m-s)+N_{Q}(m-s)=0, \quad s=1, \ldots,[m / 2] .
\end{gathered}
$$

Note that

$$
\begin{array}{ll}
k_{j m}=k_{j, 2 m}+k_{j+m, 2 m}, & r_{j m}=r_{j, 2 m}+r_{j+m, 2 m}, \\
p_{j m}=p_{j, 2 m}+p_{j+m, 2 m}, & q_{j m}=q_{j, 2 m}+q_{j+m, 2 m},
\end{array} \quad j=1, \ldots, m .
$$

From (9) we can find further restrictions for $k_{i m}, r_{i m}, p_{i m}, q_{i m}$, as noted in the following theorem.

Theorem 3. If $A, B, C, D$ are $\mathrm{BS}(2 n+1)$, then for a given $m \in\{2,3, \ldots$, $n+1\}$,

$$
\begin{aligned}
& k_{1 m}+r_{1 m}+k_{n+1, m}+r_{n+1, m} \equiv\left\{\begin{array}{l}
2 \bmod 4 \text { if } n \neq 0 \bmod m, \\
0 \bmod 4 \text { if } n \equiv 0 \bmod m,
\end{array}\right. \\
& k_{j m}+r_{j m}+k_{n+2-j, m}+r_{n+2-j, m} \equiv 0 \bmod 4, \quad j=2, \ldots, m, \\
& p_{j m}+q_{j m}+p_{n+1-j, m}+q_{n+1-j, m} \equiv 0 \bmod 4, \quad j=1, \ldots, m,
\end{aligned}
$$

where $k_{i m}, r_{i m}, p_{i m}, q_{i m}$ are defined in (27).

Proof. Summing all relations in (9) with $s \equiv j \bmod m$, we obtain the above result.

The Algorithm. Since it is difficult to find directly the values of $a_{1}, \ldots, a_{n+1}$, $b_{1}, \ldots, b_{n+1}, c_{1}, \ldots, c_{n}, d_{1}, \ldots, d_{n}$, we find the values of

$$
k_{1 m}, \ldots, k_{m m}, r_{1 m}, \ldots, r_{m m}, p_{1 m}, \ldots, p_{m m}, q_{1 m}, \ldots, q_{m m}
$$

as defined in (27). Our algorithm relies on Corollary 1. To avoid calculating isomorphic $\mathrm{BS}(2 n+1)$, on the basis of the four properties given in (26), we can always take

$$
k_{11} \geq r_{11} \geq 0, \quad p_{11} \geq q_{11} \geq 0
$$

where $k_{11}, r_{11}, p_{11}, q_{11}$ is the sum of the elements of $A, B, C, D$, respectively. If $n$ is odd, then $k_{12} \geq k_{22} \geq 0, r_{12} \geq r_{22}$. If $n$ is even, then $p_{12} \geq p_{22} \geq 0, q_{12} \geq q_{22}$.

Step 1. Find all $k_{11}, r_{11}, p_{11}, q_{11}$ satisfying

$$
k_{11} \geq r_{11} \geq 0, \quad p_{11} \geq q_{11} \geq 0, \quad k_{11}^{2}+r_{11}^{2}+p_{11}^{2}+q_{11}^{2}=4 n+2,
$$

and for

(i) $n$ even, take $k_{11}, r_{11}$ odd,

(ii) $n$ odd, take $k_{11}, q_{11}$ even,

$p_{11}, q_{11}$ odd. 
Step 2. For every quadruple $k_{11}, r_{11}, p_{11}, q_{11}$ and given $m \in\{2,3, \ldots$, $n+1\}$, find $k_{1 m}, \ldots, k_{m m}, r_{1 m}, \ldots, r_{m m}, p_{1 m}, \ldots, p_{m m}, q_{1 m}, \ldots, q_{m m}$ satisfying

(i) $k_{11}=k_{1 m}+\cdots+k_{m m}, r_{11}=r_{1 m}+\cdots+r_{m m}, p_{11}=p_{1 m}+\cdots+p_{m m}$, $q_{11}=q_{1 m}+\cdots+q_{m m}$

(ii) $k_{j m}, r_{j m}$ are both odd (even) if $[(n+1-j) / m]+1$ is odd (even) and $\left|k_{j m}\right| \leq\left[\frac{n+1-j}{m}\right]+1, \quad\left|r_{j m}\right| \leq\left[\frac{n+1-j}{m}\right]+1, \quad j=1, \ldots, m$, $p_{j m}, q_{j m}$ are both odd (even) if $[(n-j) / m]+1$ is odd (even) and $\left|p_{j m}\right| \leq\left[\frac{n-j}{m}\right]+1, \quad\left|q_{j m}\right| \leq\left[\frac{n-j}{m}\right]+1, \quad j=1, \ldots, m$,

(iii)

$$
\begin{aligned}
& k_{1 m}+r_{1 m}+k_{n+1, m}+r_{n+1, m} \equiv\left\{\begin{array}{l}
2 \bmod 4 \text { if } n \neq 0 \bmod m, \\
0 \bmod 4 \text { if } n \equiv 0 \bmod m,
\end{array}\right. \\
& k_{j m}+r_{j m}+k_{n+2-j, m}+r_{n+2-j, m} \equiv 0 \bmod 4, \quad j=2, \ldots, m, \\
& p_{j m}+q_{j m}+p_{n+1-j, m}+q_{n+1-j, m} \equiv 0 \bmod 4, \quad j=1, \ldots, m,
\end{aligned}
$$

(iv) $k_{1 m}^{2}+\cdots+k_{m m}^{2}+r_{1 m}^{2}+\cdots+r_{m m}^{2}+p_{1 m}^{2}+\cdots+p_{m m}^{2}+q_{1 m}^{2}+\cdots+q_{m m}^{2}=4 n+2$,

(v) $N_{K}(s)+N_{K}(m-s)+N_{R}(s)+N_{R}(m-s)+N_{P}(s)+N_{P}(m-s)+N_{Q}(s)+$ $N_{Q}(m-s)=0, s=1, \ldots,[m / 2]$, where

$$
\begin{array}{ll}
N_{K}(s)=\sum_{j=1}^{m-s} k_{j m} k_{j+s, m}, & N_{R}(s)=\sum_{j=1}^{m-s} r_{j m} r_{j+s, m}, \\
N_{P}(s)=\sum_{j=1}^{m-s} p_{j m} p_{j+s, m}, & N_{Q}(s)=\sum_{j=1}^{m-s} q_{j m} q_{j+s, m} .
\end{array}
$$

Step 3. (i) For every $k_{1 m}, \ldots, k_{m m}, r_{1 m}, \ldots, r_{m m}, p_{1 m}, \ldots, p_{m m}, q_{1 m}, \ldots$, $q_{m m}$ found in Step 2, find $k_{1,2 m}, \ldots, k_{2 m, 2 m}, r_{1,2 m}, \ldots, r_{2 m, 2 m}, p_{1,2 m}, \ldots$, $p_{2 m, 2 m}, q_{1,2 m}, \ldots, q_{2 m, 2 m}$ satisfying

$$
\begin{array}{ll}
k_{j m}=k_{j, 2 m}+k_{j+m, 2 m}, & r_{j m}=r_{j, 2 m}+r_{j+m, 2 m}, \\
p_{j m}=p_{j, 2 m}+p_{j+m, 2 m}, & q_{j m}=q_{j, 2 m}+q_{j+m, 2 m},
\end{array} \quad j=1, \ldots, m .
$$

(ii) Go to Step 2(ii)-(v), setting $2 m$ instead of $m$.

Step 4. Stop when $m \geq n+1$ and examine if

$$
N_{K}(s)+N_{R}(s)+N_{P}(s)+N_{Q}(s)=0, \quad s=1, \ldots, m-1,
$$

because for $m \geq n+1$,

$$
\begin{array}{ll}
k_{j m}=0,1,-1, & r_{j m}=0,1,-1, \\
p_{j m}=0,1,-1, & q_{j m}=0,1,-1 .
\end{array}
$$




\section{TABLE 1}

Base sequences $\mathrm{BS}(2 n+1) A, B, C, D$ of lengths $n+1, n+1, n, n$ or Suitable sequences $\mathrm{SS}(2 n+1)(A+B) / 2,(A-B) / 2,(C+D) / 2,(C-D) / 2$

\begin{tabular}{|c|c|c|}
\hline Length & $\begin{array}{l}\text { Sum of Squares } \\
\text { for Suitable Sequences }\end{array}$ & Sequence \\
\hline$n=19$ & $2 n+1=39=6^{2}+1^{2}+1^{2}+1^{2}$ & 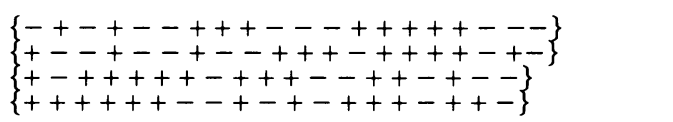 \\
\hline$n=19$ & $2 n+1=39=5^{2}+3^{2}+2^{2}+1^{2}$ & 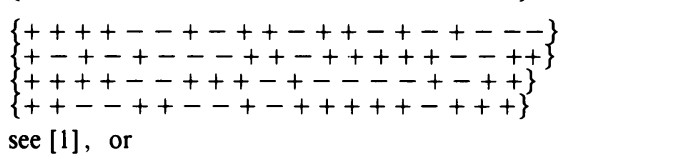 \\
\hline$n=20$ & $2 n+1=41=6^{2}+2^{2}+1^{2}+0^{2}$ & $\begin{array}{l}\left\{\begin{array}{l}++-+--+--+++--+++-+-- \\
--++-++++--+++-+--+- \\
+-+-++++++-++-----++\} \\
+++++-+++-+++-+-+--+\}\end{array}\right. \\
\text { see [7], or }\end{array}$ \\
\hline$n=20$ & $2 n+1=41=5^{2}+4^{2}+0^{2}+0^{2}$ & 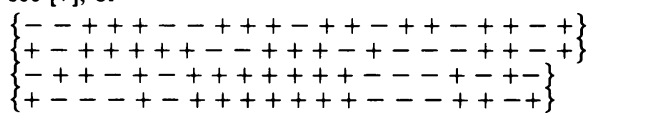 \\
\hline$n=20$ & $2 n+1=41=4^{2}+4^{2}+3^{2}+0^{2}$ & 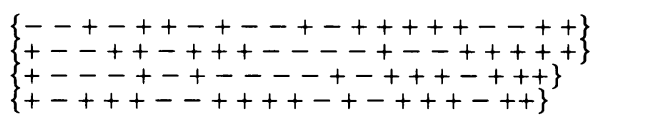 \\
\hline$n=21$ & $2 n+1=43=5^{2}+4^{2}+1^{2}+1^{2}$ & 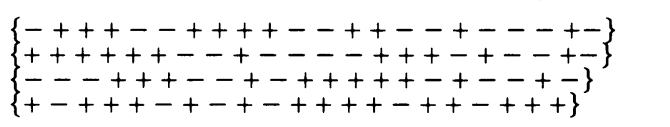 \\
\hline$n=21$ & $2 n+1=43=5^{2} \cdot 2^{2}+3^{2}+0^{2}$ & $\begin{array}{l}\left\{\begin{array}{l}+++++--+--+-+---++--+ \\
++-+++-++-+-++--++++--\} \\
-+--++++++-++-+-+++-- \\
+--++++++---+-+-+-+++\end{array}\right\} \\
\text { see [7], or }\end{array}$ \\
\hline$n=21$ & $2 n+1=43=4^{2}+3^{2}+3^{2}+3^{2}$ & 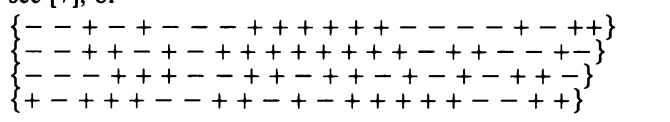 \\
\hline$n=22$ & $2 n+1=45=6^{2}+3^{2}+0^{2}+0^{2}$ & 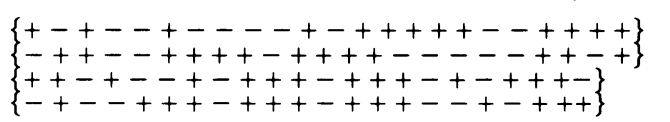 \\
\hline$n=22$ & $2 n+1=45=6^{2}+2^{2}+2^{2}+1^{2}$ & 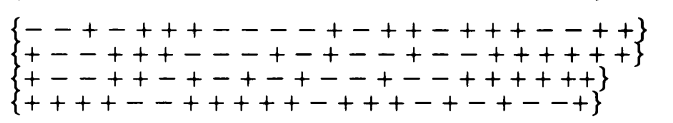 \\
\hline$n=22$ & $2 n+1=45=5^{2}+4^{2}+2^{2}+0^{2}$ & $\begin{array}{l}\left\{\begin{array}{l}\{+-+--+++++++---++++-+ \\
+-+-+-+-1+---+++++++-++ \\
+-+-++---1++--++-+++- \\
+-++--+++-+++++--+-++-\}\end{array}\right\} \\
\text { see [7], or }\end{array}$ \\
\hline$n=22$ & $2 n+1=45=4^{2}+4^{2}+3^{2}+2^{2}$ & 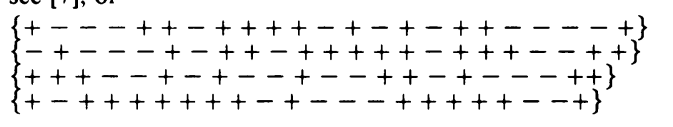 \\
\hline$n=23$ & $2 n+1=47=6^{2}+3^{2}+1^{2}+1^{2}$ & 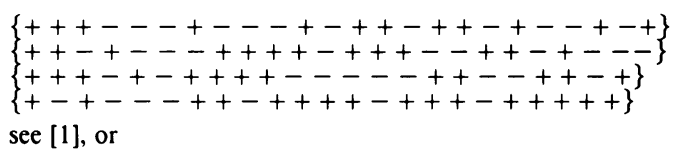 \\
\hline$n=23$ & $2 n+1=47=5^{2}+3^{2}+3^{2}+2^{2}$ & 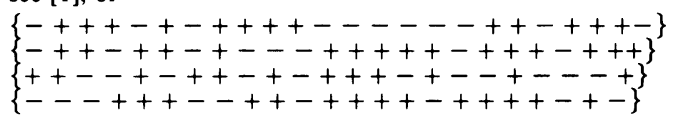 \\
\hline
\end{tabular}


TABLE 1 (continued)

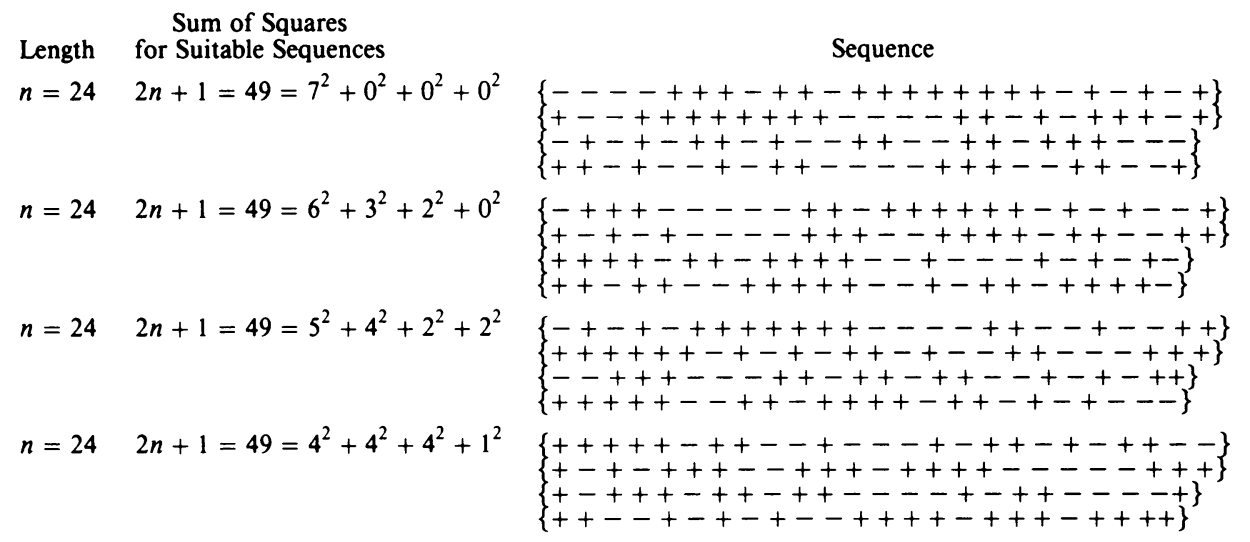

Remark. C. H. Yang and J. Yang (private communication) have constructed the equivalent of the sequences shown in Table 1 by constructing base, normal, or near normal sequences.

\section{TURYN SEQUENCES}

Our algorithm requires too much computer time for the construction of $\operatorname{BS}(2 n+1)$ for $n \geq 25$. It is desirable to construct $\mathrm{BS}(4 n+3)$ from $\mathrm{BS}(2 n+1)$.

Theorem 4. If $A, B, C, D$ are $\mathrm{BS}(2 n+1)$, and their associated polynomials satisfy

$$
A(z) C\left(z^{-1}\right)+z C(z) A\left(z^{-1}\right)=0, \quad z \neq 0,
$$

then the sequences

$$
X=\{1, A \mid C\}, \quad Y=\{-1, A \mid C\}, \quad Z=\{B \mid D\}, \quad W=\{B \mid-D\}
$$

are $\mathrm{BS}(4 n+3)$, where

$$
\begin{aligned}
& A \mid C=\left\{a_{1}, c_{1}, a_{2}, c_{2}, \ldots, a_{n}, c_{n}, a_{n+1}\right\}, \\
& B \mid D=\left\{b_{1}, d_{1}, b_{2}, d_{2}, \ldots, b_{n}, d_{n}, b_{n+1}\right\} .
\end{aligned}
$$

Proof. The polynomials associated with $X, Y, Z, W$ are

$$
\begin{aligned}
& X(z)=1+z\left(A\left(z^{2}\right)+z C\left(z^{2}\right)\right), \quad Y(z)=-1+z\left(A\left(z^{2}\right)+z C\left(z^{2}\right)\right), \\
& Z(z)=B\left(z^{2}\right)+z D\left(z^{2}\right), \quad W(z)=B\left(z^{2}\right)-z D\left(z^{2}\right) .
\end{aligned}
$$

Then

$$
\begin{aligned}
X(z) X & \left(z^{-1}\right)+Y(z) Y\left(z^{-1}\right)+Z(z) Z\left(z^{-1}\right)+W(z) W\left(z^{-1}\right) \\
= & 2+2\left(A\left(z^{2}\right) A\left(z^{-2}\right)+B\left(z^{2}\right) B\left(z^{-2}\right)+C\left(z^{2}\right) C\left(z^{-2}\right)+D\left(z^{2}\right) D\left(z^{-2}\right)\right) \\
& +2 z^{-1}\left(z^{2} C\left(z^{2}\right) A\left(z^{-2}\right)+A\left(z^{2}\right) C\left(z^{-2}\right)\right)=8 n+6, \quad z \neq 0 .
\end{aligned}
$$


If we define the cross correlations

$$
N_{A C}(s)=\sum_{i=1}^{n-s} a_{i} c_{i+s}, \quad N_{C A}(s)=\sum_{i=1}^{n+1-s} c_{i} a_{i+s}, \quad s=0,1, \ldots, n,
$$

then

$$
C(z) A\left(z^{-1}\right)=\sum_{s=0}^{n} N_{A C}(s) z^{s}+\sum_{s=1}^{n} N_{C A}(s) z^{-s}
$$

and

$$
z C(z) A\left(z^{-1}\right)+A(z) C\left(z^{-1}\right)=\sum_{s=1}^{n}\left(N_{A C}(s-1)+N_{C A}(s)\right)\left(z^{s}+z^{-s+1}\right) .
$$

Therefore, $z C(z) A\left(z^{-1}\right)+A(z) C\left(z^{-1}\right)=0$ is equivalent to

$$
N_{A C}(s-1)+N_{C A}(s)=0, \quad s=1, \ldots, n .
$$

Definition. If the sequences $A, B, C, D$ are $\mathrm{BS}(2 n+1)$ and satisfy

$$
N_{A C}(s-1)+N_{C A}(s)=0, \quad s=1, \ldots, n,
$$

then they are called Turyn sequences (abbreviated as $\operatorname{TS}(2 n+1)$ ).

Theorem 5 (see also [3, pp. 139-142]). If $A, B, C, D$ are $\operatorname{TS}(2 n+1)$, then

(i) For $n$ odd,

$$
\begin{aligned}
& A=\left\{A_{1},-A_{1}^{*}\right\}, \quad B=\left\{b_{1}, B_{1},-B_{1}^{*}, b_{1}\right\}, \\
& C=\left\{C_{1}, c_{(n+1) / 2}, C_{1}^{*}\right\}, \quad D=\left\{D_{1}, d_{(n+1) / 2}, D_{1}^{*}\right\},
\end{aligned}
$$

where

$$
\begin{array}{ll}
A_{1}=\left\{a_{1}, \ldots, a_{(n+1) / 2}\right\}, & B_{1}=\left\{b_{2}, \ldots, b_{(n+1) / 2}\right\}, \\
C_{1}=\left\{c_{1}, \ldots, c_{(n-1) / 2}\right\}, & D_{1}=\left\{d_{1}, \ldots, d_{(n-1) / 2}\right\} .
\end{array}
$$

(ii) For $n$ even,

$$
\begin{gathered}
A=\left\{A_{1}, a_{n / 2+1}, A_{1}^{*}\right\}, \quad B=\left\{b_{1}, B_{1}, b_{n / 2+1}, B_{1}^{*},-b_{1}\right\}, \\
C=\left\{C_{1},-C_{1}^{*}\right\}, \quad D=\left\{D_{1},-D_{1}^{*}\right\},
\end{gathered}
$$

where

$$
\begin{array}{ll}
A_{1}=\left\{a_{1}, \ldots, a_{n / 2}\right\}, & B_{1}=\left\{b_{2}, \ldots, b_{n / 2}\right\}, \\
C_{1}=\left\{c_{1}, \ldots, c_{n / 2}\right\}, & D_{1}=\left\{d_{1}, \ldots, d_{n / 2}\right\} .
\end{array}
$$

Proof. From (38) and from $x \cdot y \equiv(x+y-1) \bmod 4$ when $x, y= \pm 1$, we have

$$
F(s):=\sum_{i=1}^{n+1-s} a_{i}+\sum_{i=s+1}^{n+1} a_{i}+\sum_{i=1}^{n+1-s} c_{i}+\sum_{i=s}^{n} c_{i} \equiv 2(n+1-s) \bmod 4, \quad s=1, \ldots, n
$$


for $s=n+1,(37)$ gives

$$
a_{1}+a_{n+1}+c_{1}+c_{n} \equiv 2 \bmod 4 .
$$

Then

$$
\begin{aligned}
F(s-1)-F(s)=a_{n+2-s}+a_{s}+c_{n+2-s}+c_{s-1} \equiv 2 \bmod 4, & \\
& s=2, \ldots, n+1 .
\end{aligned}
$$

Proceeding as in Theorem 1, we obtain from (39) and (9)

(i) For $n$ odd:

$$
\begin{aligned}
& a_{s}+a_{n+2-s} \equiv 0 \bmod 4, \quad s=1, \ldots, n+1, \\
& c_{s}+c_{n+1-s} \equiv 2 \bmod 4, \quad s=1, \ldots, n \text {, } \\
& b_{s}+b_{n+2-s} \equiv \begin{cases}2 \bmod 4, & s=1, n+1, \\
0 \bmod 4, & s=2, \ldots, n .\end{cases}
\end{aligned}
$$

(ii) For $n$ even:

$$
\begin{aligned}
& a_{s}+a_{n+2-s} \equiv 2 \bmod 4, \quad s=1, \ldots, n+1, \\
& b_{s}+b_{n+2-s} \equiv \begin{cases}0 \bmod 4, & s=1, n+1, \\
2 \bmod 4, & s=2, \ldots, n,\end{cases} \\
& c_{s}+c_{n+1-s} \equiv 0 \bmod 4, \quad s=1, \ldots, n \text {, } \\
& d_{s}+d_{n+1-s} \equiv 0 \bmod 4, \quad s=1, \ldots, n,
\end{aligned}
$$

which proves Theorem 5 .

Our algorithm in $\S 2$ can now be modified to give $\operatorname{TS}(2 n+1)$. From Theorem 5 we have:

(i) For $n$ odd:

$$
\begin{array}{ll}
k_{j m}=-k_{n+2-j, m}, & j=1, \ldots, m, \\
r_{1 m}+r_{n+1, m}=2, & \\
r_{j m}=-r_{n+2-j, m}, & j=2, \ldots, m, \\
p_{j m}=p_{n+1-j, m}, & j=1, \ldots, m, \\
q_{j m}=q_{n+1-j, m}, & j=1, \ldots, m .
\end{array}
$$

(ii) For $n$ even:

$$
\begin{array}{ll}
k_{j m}=k_{n+2-j, m}, & j=1, \ldots, m, \\
r_{j m}=r_{n+2-j, m}, & j=2, \ldots, m, \\
r_{1 m}-r_{n+1, m}=2 & \text { for } n \not \equiv 0 \bmod 4, \\
p_{j m}=-p_{n+1-j, m}, & j=1, \ldots, m, \\
q_{j m}=-q_{n+1-j, m}, & j=1, \ldots, m .
\end{array}
$$


It is known that $\operatorname{TS}(2 n+1)$ exists for $n \leq 7$ and $n=12,14$; they cannot exist for $n=10,11,16,17$, and for $n=8,9,13,15, \operatorname{TM}(2 n+1)$ might exist but an exhaustive machine search showed that they do not exist (see $[1 ; 3$, pp. 142-143]).

In this paper, applying our algorithm, we have done an exhaustive search, and we showed that $\operatorname{TS}(2 n+1)$ does not exist for $n=18,19, \ldots, 27$; of course, for $n=28,29, \operatorname{TS}(2 n+1)$ cannot exist.

\section{ACKNOWLEDGMENTS}

We thank the referee for his thoughtful comments.

\section{BIBLIOGRAPHY}

1. G. Cohen, D. Rubie, J. Seberry, C. Koukouvinos, S. Kounias, and M. Yamada, A survey of base sequences, disjoint complementary sequences and $O D(4 t ; t, t, t, t), \mathrm{J}$. Combin. Math. and Combin. Computing 5 (1989), 60-104.

2. J. Cooper and J. Wallis, A construction for Hadamard arrays, Bull. Australian Math. Soc. 7 (1972), 269-277.

3. A. V. Geramita and J. Seberry, Orthogonal designs: Quadratic forms and Hadamard matrices, Marcel Dekker, New York-Basel, 1979.

4. C. Koukouvinos, S. Kounias, and J. Seberry, Further results on base sequences, disjoint complementary sequences, $O D(4 t ; t, t, t, t)$ and the excess of Hadamard matrices, Ars Combin. (to appear).

5. R. J. Turyn, Hadamard matrices, Baumert-Hall units, four-symbol sequences, pulse compressions and surface wave encodings, J. Combin. Theory Ser. A 16 (1974), 313-333.

6. C. H. Yang, A composition theorem for $\delta$-codes, Proc. Amer. Math. Soc. 89 (1983), 375-378.

7 . On composition of four-symbol $\delta$-codes and Hadamard matrices, Proc. Amer. Math. Soc. 107 (1989), 763-776.

Department of Mathematics, University of Thessaloniki, Thessaloniki 54006, Greece (Koukouvinos and Sotirakoglou)

Department of Mathematics, University of Athens, Panepistemiopolis 157 84, Greece (Kounias) 